\title{
Risk factors predicting a higher grade of subarachnoid haemorrhage in small ruptured intracranial aneurysm $(<5 \mathrm{~mm})$
}

\author{
Junli Tai, Jianxin Liu, Jianhua Lv, Kang Huibin, Zhe Hou, Jun Yang, Hongbing Zhang, Qing Huang \\ Department of Neurosurgery in Beijing Luhe Hospital, Capital Medical University, Beijing, China
}

\begin{abstract}
Aim. To identify the risk factors for clinical and radiographic grades of subarachnoid haemorrhage (SAH) in small $(<5 \mathrm{~mm})$ intracranial aneurysms (SIAs).

Material and methods. We retrospectively analysed patients with SIAs treated in our centre between February 2009 and June 2018. The clinical status was graded using the Hunt and Hess (H\&H) score and the radiological severity of SAH was graded by Fisher grades $(\mathrm{FG})$. The risk factors were determined using multivariate logistic regression analysis.

Results. A total of 160 patients with ruptured SIAs $(<5 \mathrm{~mm})$ were included. In univariate analysis, smoking $(P=0.007)$, alcohol use $(P=0.048)$, aspirin use $(P=0.001)$, and higher size ratio $(S R)(P=0.001)$ were significantly associated with a higher $H \& H$ grade (3-5) in SIAs; and smoking $(P=0.019)$, aspirin use $(P=0.031)$, inflow angle $<90$ degrees $(P=0.011)$, and aneurysm size $(P=0.039)$ were significantly associated with a higher $\mathrm{FG}$ score (3-4). In the adjusted multivariate analysis, previous $\mathrm{SAH}(\mathrm{OR}, 12.245,95 \% \mathrm{Cl}$, 2.261-66.334, $\mathrm{P}=0.004)$, aspirin use $(\mathrm{OR}, 4.677,95 \% \mathrm{Cl}, 1.392-15.718, \mathrm{P}=0.013)$, alcohol use $(\mathrm{OR}, 3.392,95 \% \mathrm{Cl}, 1.146-10.045, \mathrm{P}$ $=0.027)$, inflow angle $<90(\mathrm{OR}, 3.881,95 \% \mathrm{Cl}, 1.273-11.831, \mathrm{P}=0.017)$, and higher $\mathrm{SR}(\mathrm{OR}, 6.611,95 \% \mathrm{Cl}, 2.235-19.560, \mathrm{P}=0.001)$ were independent risk factors for a higher $\mathrm{H} \& \mathrm{H}$ grade in ruptured SIAs; smoking $(\mathrm{OR}, 2.157,95 \% \mathrm{Cl}, 1.061-4.384, \mathrm{P}=0.034)$, and inflow angle $<90$ degrees $(\mathrm{OR}, 2.603,95 \% \mathrm{Cl}, 1.324-5.115, \mathrm{P}=0.006)$ were independent risk factors for a higher $\mathrm{FG}(3-4)$.

Conclusions. This study revealed that inflow angle $<90$ degrees and size ratio, but not absolute size, may highly predict poorer grade of SAH in SRA. Aspirin use, previous $\mathrm{SAH}$, and alcohol use were significantly associated with a higher $\mathrm{H} \& \mathrm{H}$ grade in ruptured SIAs, and smoking was a significant predictor of poorer FG.
\end{abstract}

Key words: intracranial aneurysm, small, rupture, aspirin use, Hunt and Hess score, Fisher grade

(Neurol Neurochir Pol 2019; 53 (4): 296-303)

\section{Introduction}

With a 30 -day mortality rate of $40 \%$, the most serious damage caused by intracranial aneurysms (IAs) is subarachnoid haemorrhage (SAH) [1-3]. The rupture risk of IAs has been known to increase with increasing aneurysm size. However, some authors have found that the incidence of SAH caused by small aneurysms was increased [4-7], and have clearly demonstrated that approximately $13-50 \%$ of ruptured IAs (RIAs) were small $(<5 \mathrm{~mm})[8,9]$. As with large aneurysms, the most widely used scales for grading the disease severity of SAH in small $(<5 \mathrm{~mm})$ intracranial aneurysms (SIAs) are the Hunt and Hess $(\mathrm{H} \& \mathrm{H})$ and the Fisher grades (FG). Many articles have indicated that small aneurysms have different morphological characteristics; however, whether these characteristics are related to the disease severity of SAH is still unclear.
There is a plethora of available literature reporting metabolic indicators as potential markers of the disease severity of an SAH $[10,11]$. Although different morphologic parameters such as aspect ratio (AR), size ratio (SR), and inflow angle have been believed to contribute significantly to determining the risk of small aneurysm rupture [9, 12-14], the impact of these morphologic features on the severity of SAH caused by an SRA still remains unexplored.

In addition, antiplatelet agents (aspirin in particular) have emerged as possible options for noninvasive treatment of IAs to decrease the incidence of aneurysmal SAH. On the other hand, according to a meta-analysis of seven studies, aspirin use may be significantly associated with an increased risk of aneurysm rupture [15]. However, the association between aspirin use and the clinical and radiographic grades of SAH caused by SIAs remains unclear. 
For small aneurysms $(<5 \mathrm{~mm})$ that are often considered to be at a low risk of rupture, conservative treatment is generally recommended clinically $[8,9,12]$, so small aneurysms are the most likely type of aneurysm to receive conservative observations and protective treatments such as taking aspirin. Therefore, it is important to investigate the risk factors affecting the severity of SAH caused by small aneurysms.

In this study, we aimed to investigate the effects of morphologic parameters and history of aspirin use on clinical and radiographic grades of SAH caused by SIAs.

\section{Material and methods}

A total of 160 patients (84 women and 76 men) with ruptured small IAs (SIA) $(<5 \mathrm{~mm})$ who were diagnosed and treated between February 2009 and June 2018 at our centre were retrospectively analysed in this study. The exclusion criteria were as follows: 1) fusiform, mycotic, and traumatic aneurysms; 2) aneurysm diameter $\geq 5 \mathrm{~mm}$; 3 ) intracranial haemorrhage due to unknown reasons, or inability to identify the location of the ruptured aneurysm among multiple IAs; 4) inability to evaluate aneurysm geometry and morphology with computed tomography angiography/ /digital subtraction angiography (CTA/DSA); and 5) patients who presented other cerebrovascular diseases such as cerebral arteriovenous malformation, arteriovenous fistula, or moyamoya disease.

\section{Definition of variables and data collection}

The patients' neurologic statuses were established based on retrospective analyses of their medical records. The severity of SAH was assessed with the Fisher Grade (FG) based on computed tomography (CT) imaging, and with the Hunt and Hess (H\&H) Grade based on clinical presentation.

Patient-specific characteristics collected in the study are shown in Table 1. Heart comorbidities were defined as coronary heart disease, heart valve disease, arrhythmias, and heart dysfunction. Cerebral ischaemic comorbidities were defined as cerebral infarction, transient ischaemic attack, and cerebral vascular stenosis, coronary artery bypass grafting, and percutaneous transluminal coronary arthroplasty. In addition, we collected data on aspirin and statins use (excluding patients on dual antiplatelet agents or non-aspirin antiplatelet agents) at the time of diagnosis of the intracranial aneurysms.

The following morphometric measurements of the aneurysmal characteristics were taken: bifurcation aneurysms (yes/no), multiple IAs (yes/no), irregular shape (yes/no), and location (internal carotid artery rather than the posterior communicating artery/posterior communicating artery/anterior communicating artery/middle cerebral artery/posterior circulation). We also collected the following variables: the size of the aneurysm, the aspect ratio (AR; dome-to-neck ratio), the size ratio (SR), and the inflow angle ( $\geq 90$ degrees $/<90$ degrees).
Table 1. Basic characteristics of all patients and SIAs

\begin{tabular}{|c|c|}
\hline & $\mathbf{N}(\%)$ \\
\hline No. & 160 \\
\hline Female & $83(51.9)$ \\
\hline Age < 50 (years) & $69(43.1)$ \\
\hline Hypertension & $81(50.6)$ \\
\hline Diabetes mellitus & $12(7.5)$ \\
\hline Hypercholesterolemia & $10(6.3)$ \\
\hline History of stroke & $11(6.9)$ \\
\hline Previous SAH & $7(4.4)$ \\
\hline Cardiovascular diseases & $9(5.6)$ \\
\hline Smoking & $54(33.8)$ \\
\hline Alcohol use & $41(25.6)$ \\
\hline Aspirin use & $20(12.5)$ \\
\hline Statins use & $17(10.6)$ \\
\hline Inflow angle $<90$ degrees & $88(55.0)$ \\
\hline Irregular shapes & $99(69.7)$ \\
\hline Aneurysm size (<3 mm) & $49(30.6)$ \\
\hline Multiple aneurysms & $17(10.6)$ \\
\hline$A R($ mean $\pm S D)$ & $1.34 \pm 0.83$ \\
\hline$S R($ mean $\pm S D)$ & $1.70 \pm 0.77$ \\
\hline \multicolumn{2}{|l|}{ Location } \\
\hline ICA & $23(14.4)$ \\
\hline $\mathrm{ACOA}$ & $58(36.3)$ \\
\hline PCoA & $36(22.5)$ \\
\hline ACA & $12(7.5)$ \\
\hline MCA & $9(5.6)$ \\
\hline$P C$ & $22(13.8)$ \\
\hline Location of bifurcation & $122(73.6)$ \\
\hline \multicolumn{2}{|l|}{ H\&H grade } \\
\hline 0 & $3(1.9)$ \\
\hline 1 & $81(50.6)$ \\
\hline 2 & $50(31.3)$ \\
\hline 3 & $19(11.9)$ \\
\hline 4 & $6(3.8)$ \\
\hline 5 & $1(0.6)$ \\
\hline \multicolumn{2}{|l|}{ FG } \\
\hline 1 & $4(2.5)$ \\
\hline 2 & 79 (49.4) \\
\hline 3 & $51(31.9)$ \\
\hline 4 & $26(16.3)$ \\
\hline
\end{tabular}

$\mathrm{SD}$ - standard deviation; $\mathrm{AR}$ - aspect ratio; $\mathrm{SR}$ - size ratio; $\mathrm{PCOM}$ - internal carotid-posterior communicating artery; $\mathrm{ACOA}$ - anterior communicating artery; $\mathrm{MCA}$ — middle cerebral artery; ICA - internal carotid artery; $\mathrm{PC}$ - basilar tip and basilar-superior cerebellar artery AND vertebral artery-posterior inferior cerebellar artery and vertebrobasilar junction; ACA - anterior cerebral artery

Bifurcation aneurysms were defined as aneurysms located at the parent artery bifurcations (anterior communicating artery, internal carotid artery terminus, posterior communicating artery, middle cerebral artery bifurcation, and the apex of the 


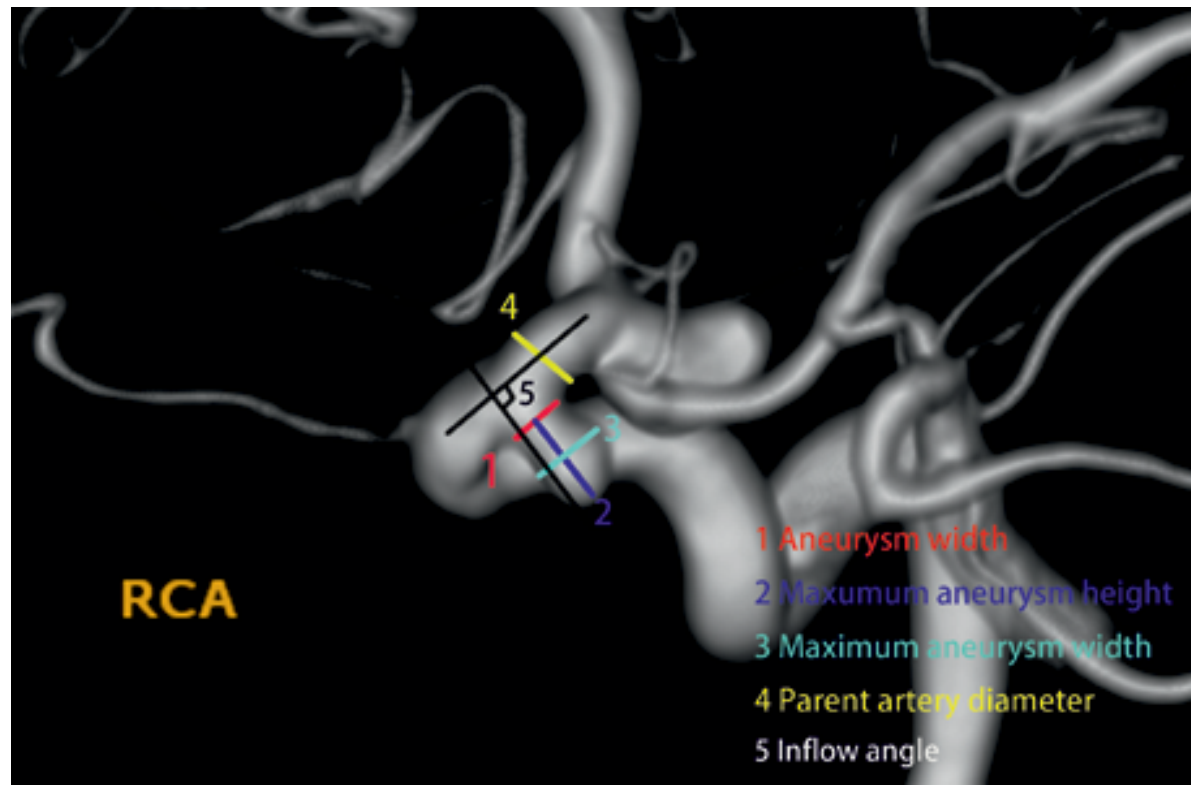

Figure 1. Aneurysm parameter definitions

basilar artery). Size ratio (SR) was defined as the maximum aneurysm height/average of the parent diameter. The aspect ratio (AR) was defined as the ratio of the maximum perpendicular height to the average neck diameter. Inflow angle was defined as the angle between the axis of flow in the parent vessel at the level of the aneurysm neck and the aneurysm's main axis from the centre of the neck to the tip of the dome (Fig. 1).

All morphological parameters were based on three-dimensional CTA/DSA imaging results and evaluated by two experienced neurosurgeons.

\section{Statistical analysis}

The data used in this study was analysed using SPSS software (SPSS 23.0, Chicago IL, USA). Data was analysed using frequencies (percentages) for categorical variables and mean \pm SD for continuous variables. Data relating to categorical variables was analysed with Fisher's exact test or the Pearson chi-square test. Data relating to continuous variables was analysed using the Mann-Whitney U test or Student's $t$-test. Unconditional logistic regression analysis was used to calculate univariate and multivariate odds ratios (ORs) with $95 \%$ confidence intervals (CI). A P-value $<0.05$ was regarded as statistically significant.

\section{Results}

\section{Study population}

A total of 160 patients (84 women and 76 men) with ruptured SIAs $(<5 \mathrm{~mm})$ were retrospectively analysed in this study. Thirty-two patients were excluded for the following reasons: inability to identify the location of the ruptured aneurysm in multiple IAs $(\mathrm{n}=17)$; dissecting, fusiform or traumatic aneurysm $(n=10)$; or aneurysms that were related to a cerebral arteriovenous malformation, arteriovenous fistula, or moyamoya disease $(n=5)$. Baseline characteristics of all patients and SIAs are shown in Table 1.

\section{Univariate analyses}

In univariate analysis, the following covariates met our previously determined level of significance and entered the forward stepwise selection for the unconditional logistic model: previous SAHs $(P<0.006)$, smoking $(P=0.007)$, alcohol use $(P=0.048)$, aspirin use $(P=0.001)$, inflow angle $<90$ degrees $(P=0.053)$, aneurysm size $(\geq 3 \mathrm{~mm},<5 \mathrm{~mm})(P=0.102)$, and a higher SR $(P=0.001)$ (Tab. 2$)$.

Factors that were significantly associated with a higher FG (3-4) were smoking $(P=0.019)$, aspirin use $(P=0.031)$, inflow angle $<90$ degrees $(P=0.011)$, and aneurysm size $(\geq$ $3 \mathrm{~mm},<5 \mathrm{~m})(P=0.039)$ (Tab. 3).

\section{Multivariate analyses}

In the final adjusted multivariate analysis, the following covariates met our previously determined level of significance and entered the forward stepwise selection for the unconditional logistic model: previous SAH (OR, 12.245, 95\% CI, 2.261-66.334, $P=0.004)$, aspirin use (OR, 4.677, 95\% CI, 1.392-15.718, $P=0.013)$, alcohol use (OR, 3.392, 95\% CI, 1.146-10.045, $P=0.027$ ), inflow angle $<90$ (OR, 3.881, 95\% CI, 1.273-11.831, $P=0.017)$, and higher SR (OR, 6.611, 95\% CI, 2.235-19.560, $P=0.001$ ) were independent risk factors for a higher $\mathrm{H} \& \mathrm{H}$ grade in ruptured SIAs (Tab. 2).

In the final adjusted multivariate analysis, smoking (OR, 2.157, 95\% CI, 1.061-4.384, $P=0.034)$, and inflow angle < 90 (OR, 2.603, 95\% CI, 1.324-5.115, $P=0.006$ ) were independent risk factors for a higher FG (3-4) (Tab. 3). 


\begin{tabular}{|c|c|c|c|c|c|}
\hline \multirow{2}{*}{ Characteristic } & \multirow[t]{2}{*}{ H\&H 1-2 } & \multirow[t]{2}{*}{ H\&H 3-5 } & \multirow{2}{*}{$\begin{array}{c}\text { Univariate } \\
\text { analysis } \\
\text { P }\end{array}$} & \multicolumn{2}{|c|}{ Multivariate analysis } \\
\hline & & & & OR & $\mathbf{P}$ \\
\hline Female & $74(55.2)$ & $9(34.6)$ & 0.085 & & \\
\hline Age $<50$ (years) & $60(44.8)$ & $14(53.8)$ & 0.520 & & \\
\hline Hypertension & $67(50.4)$ & $14(53.8)$ & 0.832 & & \\
\hline Diabetes mellitus & $10(7.5)$ & $2(7.7)$ & 1.000 & & \\
\hline History of stroke & $9(6.8)$ & $2(7.7)$ & 0.146 & & \\
\hline Previous SAH & $4(3.0)$ & $5(19.2)$ & 0.006 & $12.245(2.261-66.334)$ & 0.004 \\
\hline Cardiovascular diseases & $7(5.3)$ & $1(3.8)$ & 1.000 & & \\
\hline Smoking & $39(29.1)$ & $15(57.7)$ & 0.007 & & \\
\hline Alcohol use & $30(22.4)$ & $11(42.3)$ & 0.048 & $3.392(1.146-10.045)$ & 0.027 \\
\hline Aspirin use & $11(8.2)$ & $9(34.5)$ & 0.001 & $4.677(1.392-15.718)$ & 0.013 \\
\hline Statins use & $12(9.0)$ & $5(19.2)$ & 0.133 & & \\
\hline Inflow angle $<90$ degrees & $69(51.5)$ & $19(73.1)$ & 0.053 & $3.881(1.273-11.831)$ & 0.017 \\
\hline Irregular shapes & $94(70.7)$ & $20(76.9)$ & 0.637 & & \\
\hline Aneurysm size $(\geq 3 \mathrm{~mm},<5 \mathrm{~mm})$ & $89(66.4)$ & $22(84.6)$ & 0.102 & & \\
\hline Multiple aneurysms & $15(11.2)$ & $2(7.7)$ & 1.000 & & \\
\hline $\mathrm{AR}($ mean $\pm \mathrm{SD})$ & $1.37 \pm 0.85$ & $1.18 \pm 0.70$ & 0.287 & & \\
\hline $\mathrm{SR}($ mean $\pm \mathrm{SD})$ & $1.69 \pm 0.75$ & $2.22 \pm 0.78$ & 0.001 & $6.611(2.235-19.560)$ & 0.001 \\
\hline \multicolumn{6}{|l|}{ Location } \\
\hline ICA & $20(14.9)$ & $3(11.5)$ & 0.696 & & \\
\hline $\mathrm{ACoA}$ & $47(35.1)$ & $11(42.3)$ & & & \\
\hline PCoA & $30(22.4)$ & $6(23.1)$ & & & \\
\hline ACA & $9(6.7)$ & $3(11.5)$ & & & \\
\hline MCA & $9(6.7)$ & $0(0)$ & & & \\
\hline PC & $19(14.2)$ & $3(11.5)$ & & & \\
\hline Bifurcation & $101(75.0)$ & $21(80.8)$ & 0.800 & & \\
\hline
\end{tabular}

\section{Discussion}

In this study, the main finding was that inflow angle affected both the higher $\mathrm{H} \& \mathrm{H}$ grade (3-5) and the FG (3-4). In addition, aspirin use, previous $\mathrm{SAH}$, alcohol use, and higher SR were independent risk factors for a higher $\mathrm{H} \& \mathrm{H}$ grade in ruptured SIAs. Smoking was an independent risk factor for a higher FG (3-4). Therefore, the clinical management of small $(<5 \mathrm{~mm})$ unruptured IAs with these underlying factors should be more comprehensive and should be carried out more cautiously.

Inflammation plays a major role in the formation, progress, and rupture of IAs; several anti-inflammatory drugs have been tested for their potential to decrease IA formation and rupture [14, 16-19]. Aspirin has emerged as the most promising medical therapy for decreasing the incidence of aneurysmal subarachnoid haemorrhage by counteracting proinflammatory pathways and stabilising aneurysmal walls [17, 18]. Several population-based studies that have studied the association between antiplatelet therapy and subarachnoid haemorrhage (SAH) have had conflicting results. The International Study of Unruptured Intracranial Aneurysms (ISUIA) investigators reported that patients who used aspirin at least three times weekly had significantly lower risks of aneurysm rupture than those who never used aspirin, suggesting that frequent aspirin use may have a protective effect on blood vessels [20]. Along similar lines, Garcia Rodriguez et al., using data from the health improvement network, reported a lower risk of SAH with long-term aspirin intake [19]. In contrast, the use of low-dose aspirin has been associated with an increased risk of SAH in the first month after starting treatment [21]. A recent meta-analysis of seven studies suggests an increased risk of aSAH among short-term ( $<3$ months $)$ aspirin users [15]. The exact mechanism by which aspirin may exert its effects on aneurysm prognosis and rupture is unclear. Recent studies have shown that aspirin (e.g. acetylsalicylic acid) may stabilise aneurysm walls and counteract proinflammatory pathways [14, 22]. In addition, walls of ruptured IAs have higher levels of cyclooxygenase- 2 and microsomal prostaglandin $\mathrm{E} 2$ synthase 1 , both of which are inhibited by aspirin [22]. However, there is little evidence to identify the effect of aspirin use on clinical 
Table 3. Univariate analysis and multivariate analysis between FRS 1-2 group and FRS 3-4 group

\begin{tabular}{|c|c|c|c|c|c|}
\hline \multirow[t]{2}{*}{ Characteristic } & \multirow[t]{2}{*}{ FRS 1-2 } & \multirow[t]{2}{*}{ FRS 3-4 } & \multirow{2}{*}{$\begin{array}{c}\text { Univariate analysis } \\
\text { P }\end{array}$} & \multicolumn{2}{|c|}{ Multivariate analysis } \\
\hline & & & & OR & $\mathbf{P}$ \\
\hline Female & $47(56.0)$ & $36(47.4)$ & 0.342 & & \\
\hline Age $<50$ (years) & $44(52.4)$ & $42(55.3)$ & 0.752 & & \\
\hline Hypertension & $39(47.0)$ & $42(55.3)$ & 0.832 & & \\
\hline Diabetes mellitus & $6(7.1)$ & $6(8.0)$ & 1.000 & & \\
\hline History of stroke & $4(4.8)$ & $7(9.3)$ & 0.351 & & \\
\hline Previous SAH & $3(3.6)$ & $6(7.9)$ & 0.312 & & \\
\hline Cardiovascular diseases & $3(3.6)$ & $5(6.7)$ & 0.477 & & \\
\hline Smoking & $21(25.0)$ & $33(43.4)$ & 0.019 & $2.157(1.061-4.384)$ & 0.034 \\
\hline Alcohol use & $18(21.4)$ & $23(30.3)$ & 0.211 & & \\
\hline Aspirin use & $6(7.1)$ & $14(18.4)$ & $0.031^{\circ}$ & & \\
\hline Statins use & $6(7.1)$ & $11(14.5)$ & 0.133 & & \\
\hline Inflow angle $<90$ degrees & $38(45.2)$ & $50(65.8)$ & 0.011 & $2.603(1.324-5.115)$ & 0.006 \\
\hline Irregular shapes & $62(74.7)$ & $52(68.4)$ & 0.481 & & \\
\hline Aneurysm size $(\geq 3 \mathrm{~mm} \rrbracket<5 \mathrm{~mm})$ & $52(61.9)$ & $59(77.6)$ & 0.039 & & \\
\hline Multiple aneurysms & $9(10.7)$ & $8(10.5)$ & 1.000 & & \\
\hline $\mathrm{AR}($ mean $\pm \mathrm{SD})$ & $1.37 \pm 0.90$ & $1.31 \pm 0.74$ & 0.669 & & \\
\hline $\mathrm{SR}($ mean $\pm \mathrm{SD})$ & $1.74 \pm 0.87$ & $1.81 \pm 0.67$ & 0.585 & & \\
\hline \multicolumn{6}{|l|}{ Location } \\
\hline ICA & $16(19.0)$ & $7(9.2)$ & 0.696 & & \\
\hline $\mathrm{ACOA}$ & $30(35.7)$ & $28(36.8)$ & & & \\
\hline PCoA & $15(17.9)$ & $21(27.6)$ & & & \\
\hline ACA & $4(4.8)$ & $10(10.5)$ & & & \\
\hline MCA & $7(8.3)$ & $2(2.6)$ & & & \\
\hline PC & $12(14.3)$ & $10(13.2)$ & & & \\
\hline Bifurcation & $63(75.9)$ & $59(77.6)$ & 0.852 & & \\
\hline
\end{tabular}

and radiographic grades of SAH caused by SIAs. In our study, aspirin use was significantly associated with a higher $\mathrm{H} \& \mathrm{H}$ grade in ruptured SIAs, whereas statin use was probably not associated with a higher $\mathrm{H} \& \mathrm{H}$ grade or FG.

Ours is the first study in which aspirin has been significantly associated with higher $\mathrm{H} \& \mathrm{H}$ grades; further studies are needed to determine the safety of aspirin as an emerging potential medical therapy for the prevention of IA rupture.

In the study, we found that inflow angle affected both the higher $\mathrm{H} \& \mathrm{H}$ grade (3-5) and the FG (3-4). Baharoglu et al. evaluated maximal dimension, height-width ratio, and dome-neck aspect ratio sidewall-type aneurysms with respect to the rupture status in a cohort of 116 aneurysms in 102 patients. They found that inflow angle is a significant discriminate of rupture status in aneurysms and is associated with significantly greater peak velocities and kinetic energy transmission to the dome [23]. Tykocki et al. found that inflow angle proved to be a relevant predictor in estimating the aneurysm risk rupture of the posterior cerebral circulation [24]. Similarly, Lv et al. also reported that inflow angle was significantly different between ruptured and unruptured small posterior communicating artery aneurysms [25]. The most important finding of this study was that inflow angle was the only independent risk factor for both $\mathrm{H} \& \mathrm{H}$ grade and FG. Moreover, for simpler clinical measurements, we divided the inflow angle into $\geq 90$ degrees $/<90$ degrees. In the present study, it was observed that $71.3 \%$ of patients with poorer $\mathrm{H} \& \mathrm{H}$ (3-5) had an inflow angle $<90$ degrees, and $65.8 \%$ of patients with poorer FG (3-4) had an inflow angle $<90$ degrees. One possible explanation is that a greater inflow angle leads to an increase of the wall shear stress (WSS), spatial gradient, and results in a more direct flow into the aneurysm dome. These altered blood flow parameters in the aneurysm lumen may increase the risk of aneurysm rupture, and then if bleeding is not quickly stopped after the aneurysm rupture there would be a resultant increase in bleeding volume, and a poorer grade of $\mathrm{H} \& \mathrm{H}$ and FG [26-28].

The importance of the SR has been emphasised in many studies. As a promising new morphological parameter, the SR was first proposed by Dhar et al, when they reported that SR was associated with the rupture of IAs [7]. Several studies have shown a significant relationship between SR and the 
risk of rupture of small aneurysms. A study by Kashiwazaki in Japan revealed that SR might predict the risk of rupture in small unruptured IAs [5]. The study of Feng et al. also found that small IAs with larger SRs was associated with a greater rupture risk (OR, 2.766) [29]. However, there have been few studies on the possible mechanisms by which SR increases the risk of rupture. Markus reported that increasing SR aneurysm morphology presented multiple vortices and complex flow patterns and aneurysm luminal area was exposed to low wall shear stress increased with higher SR [30]. Complex flow, multiple vortices, and low aneurysmal wall shear stress have been associated with ruptured IAs in previous studies. These changes may lead to a higher risk of aneurysm rupture. In the present study, a higher SR was an independent risk factor for a higher H\&H grade (3-5) in ruptured SIAs (Tab. 2). For two aneurysms of the same size, a high SR indicates a small-sized parent artery. However, the threshold value of SR for predicting aneurysm rupture is unknown, although several previous studies have reported a higher SR value being related to the rupture status of IAs. We found that aneurysms located distally in the anterior cerebral artery and $\mathrm{M} 2 / 3$ of the middle cerebral artery were consistently associated with a higher SR, and that ruptured SIAs at these locations were associated with greater risks of a higher $\mathrm{H} \& \mathrm{H}$ grade. However, a high SR was not significantly associated with a high FG (3-4). A possible reason for this finding is that the diameter of the distal vessel is small or the space around the vessel is small, so the amount of bleeding is small; therefore, the CT image shows a low FG.

AR was first proposed by Ujiie et al. and was defined as aneurysm depth to aneurysm neck width. They found that AR in $88 \%$ of ruptured aneurysms was higher than 1.6 , while $56 \%$ of unruptured aneurysms had an AR lower than 1.6 [31]. A high AR was statistically more likely to have a greater low wall shear stress (WSS) area ratio that causes the dysfunction of the endothelium, which is consistent with the low flow theory [32]. The disruption and dysfunction of endothelial cells caused by non-physiological WSS is an important step in IA formation and rupture [33]. Therefore, a higher AR means a smaller neck and slower intra-aneurysmal blood flow, which reflects a higher risk of rupture. However, in our study, AR was not significantly associated with a high FG or a higher Hunt and Hess scale grade.

Female gender, history of $\mathrm{SAH}$, and cigarette smoking have all been identified as independent risk factors for aneurysm rupture in previous studies [34-36]. In our study, previous SAH was significantly associated with a high Hunt and Hess scale grade, and smoking was significantly associated with a high FG. However, previous SAH was not significantly associated with a high FG, and smoking was not significantly associated with a high Hunt and Hess scale grade. It seems that patients with a history of SAH may have a poor neurological status and do not correlate to the severity of SAH on CT imaging. In this study, alcohol use and aneurysm size $(\geq 3 \mathrm{~mm}$,
$<5 \mathrm{~m}$ ) also had inconsistent performances in the analysis of risk factors for the Hunt and Hess scale and the FG. In order to explore the reasons for this inconsistent performance, we analysed the Spearman's test of correlation between the FG and the Hunt and Hess scale. The results showed that there was a significant correlation between the Fisher grading scale and the Hunt and Hess scale $(P<0.001)$.

\section{Strengths and limitations}

The strengths of our study include the rigorous measurement of parameters by more than two experienced neurologists, therefore ensuring the representativeness and repeatability of the data. Besides, this is the first retrospective and systematic analysis of the risk factors for neurologic status and radiologic extent of subarachnoid haemorrhage (SAH) in small ruptured aneurysms $(<5 \mathrm{~mm})$. This is also the first study in which aspirin use has been significantly associated with a higher $\mathrm{H} \& \mathrm{H}$ grade in ruptured SIAs.

The data generated from this study provides some valuable information that may facilitate the evaluation of the safety of aspirin as an emerging potential medical therapy for preventing IA rupture. However, there were also several limitations to this study.

This study was retrospective in design and all patients were enrolled from a single centre, meaning there was an unavoidable choice bias. Secondly, the Hunt and Hess scale has been reported to have a poor interobserver agreement [36], so the World Federation of Neurosurgical Societies (WFNS) scale was chosen in some studies because of its predictability regarding values. Its application in the clinical environment is very common and convenient [37]. Finally, this study did not explore the effects of the Fisher grading scale and the Hunt and Hess scale on the outcome of ruptured SIAs patients; we will explore this separately in a future research paper.

\section{Conclusions}

In this study, the main finding was that two easily measurable morphological parameters, inflow angle and higher SR, were independent risk factors for the severity of SAH in SRAs. The clinical management of small aneurysms with these factors should be more cautious and comprehensive. Our findings also suggested that aspirin use was significantly associated with a higher $\mathrm{H} \& \mathrm{H}$ grade in ruptured SIAs. To ascertain whether aspirin should be instituted as a prophylactic measure in all patients with unruptured aneurysms requires a randomised controlled trial.

Conflict of interest: None declared.

Data availability statement: All relevant data is within the paper.

Ethics: The study protocol was approved by the institutional review board at our hospital. All patients gave written informed 
consent to participate, and the privacy of patients was strictly protected.

\section{References}

1. Darwazeh R, Wei M, Zhong J, et al. Significant injury of the mammillothalamic tract without injury of the corticospinal tract after aneurysmal subarachnoid hemorrhage: a retrospective diffusion tensor imaging study. World Neurosurg. 2018; 114: e624-e630, doi: 10.1016/j. wneu.2018.03.042, indexed in Pubmed: 29548966.

2. Huttunen J, Lindgren A, Kurki MI, et al. Epilepsy-associated long-term mortality after aneurysmal subarachnoid hemorrhage. Neurology. 2017; 89(3): 263-268, doi: 10.1212/WNL.0000000000004113, indexed in Pubmed: 28615425.

3. Koyanagi M, Fukuda $H$, Lo B, et al. Effect of intrathecal milrinone injection via lumbar catheter on delayed cerebral ischemia after aneurysmal subarachnoid hemorrhage. J Neurosurg. 2017; 12: 1-6.

4. Brown R, Broderick J. Unruptured intracranial aneurysms: epidemiology, natural history, management options, and familial screening. The Lancet Neurology. 2014; 13(4): 393-404, doi: 10.1016/s14744422(14)70015-8.

5. Dhar S, Tremmel M, Mocco J, et al. Morphology parameters for intracranial aneurysm rupture risk assessment. Neurosurgery. 2008; 63(2): 185-96; discussion 196, doi: 10.1227/01. NEU.0000316847.64140.81, indexed in Pubmed: 18797347.

6. International Study of Unruptured Intracranial Aneurysms Investigators. Unruptured intracranial aneurysms - risk of rupture and risks of surgical intervention. N Engl J Med. 1998; 339(24): 17251733, doi: 10.1056/NEJM199812103392401, indexed in Pubmed: 9867550.

7. Nahed BV, DiLuna ML, Morgan T, et al. Hypertension, age, and location predict rupture of small intracranial aneurysms. Neurosurgery. 2005; 57(4): 676-83; discussion 676, indexed in Pubmed: 16239879.

8. Bender MT, Wendt H, Monarch T, et al. Small aneurysms account for the majority and increasing percentage of aneurysmal subarachnoid hemorrhage: a 25-year, single institution study. Neurosurgery. 2018; 83(4): 692-699, doi: 10.1093/neuros/nyx484, indexed in Pubmed: 29029314.

9. Kashiwazaki D, Kuroda S. Sapporo SAH Study Group. Size ratio can highly predict rupture risk in intracranial small $(<5 \mathrm{~mm})$ aneurysms. Stroke. 2013; 44(8): 2169-2173, doi: 10.1161/STROKEAHA.113.001138, indexed in Pubmed: 23743979.

10. Poblete $R$, Cen $S$, Zheng $L$, et al. Serum lactic acid following aneurysmal subarachnoid hemorrhage is a marker of disease severity but is not associated with hospital outcomes. Frontiers in Neurology. 2018; 9, doi: 10.3389/fneur.2018.00593.

11. Savarraj JPJ, Parsha K, Hergenroeder GW, et al. Systematic model of peripheral inflammation after subarachnoid hemorrhage. Neurology. 2017; 88(16): 1535-1545, doi: 10.1212/WNL.0000000000003842, indexed in Pubmed: 28314864.

12. Duan Z, Li Y, Guan S, et al. Morphological parameters and anatomical locations associated with rupture status of small intracranial aneurysms. Sci Rep. 2018; 8(1): 6440, doi: 10.1038/s41598-018-24732-1, indexed in Pubmed: 29691446.

13. Rahman M, Smietana J, Hauck E, et al. Size ratio correlates with intracranial aneurysm rupture status: a prospective study. Stroke. 2010; 41(5): 916-920, doi: 10.1161/STROKEAHA.109.574244, indexed in Pubmed: 20378866.
14. White PM, Wardlaw JM. Unruptured intracranial aneurysms. J Neuroradiol. 2003; 30(5): 336-350, indexed in Pubmed: 14752379.

15. Chalouhi N, Atallah E, Jabbour P, et al. Aspirin for the prevention of intracranial aneurysm rupture. Neurosurgery. 2017; 64(CN_suppl_1): 114-118, doi: 10.1093/neuros/nyx299.

16. Phan K, Moore JM, Griessenauer CJ, et al. Aspirin and risk of subarachnoid hemorrhage: systematic review and meta-analysis. Stroke. 2017; 48(5): 1210-1217, doi: 10.1161/STROKEAHA.116.015674, indexed in Pubmed: 28341753.

17. Malik AN, Gross BA, Rosalind Lai PM, et al. Aspirin and aneurysmal subarachnoid hemorrhage. World Neurosurg. 2014; 82(6): 1127-1130, doi: 10.1016/j.wneu.2013.03.072, indexed in Pubmed: 23548847.

18. Hasan DM, Mahaney KB, Brown RD, et al. Aspirin as a promising agent for decreasing incidence of cerebral aneurysm rupture. Stroke. 2011; 42(11): 3156-3162, doi: 10.1161/STROKEAHA.111.619411, indexed in Pubmed: 21980208.

19. García-Rodríguez LA, Gaist D, Morton J, et al. Antithrombotic drugs and risk of hemorrhagic stroke in the general population. Neurology. 2013; 81(6): 566-574, doi: 10.1212/WNL.0b013e31829e6ffa, indexed in Pubmed: 23843464.

20. Hasan DM, Mahaney KB, Brown RD, et al. Aspirin as a promising agent for decreasing incidence of cerebral aneurysm rupture. Stroke. 2011; 42(11): 3156-3162, doi: 10.1161/STROKEAHA.111.619411, indexed in Pubmed: 21980208.

21. Pottegård A, García Rodríguez LA, Poulsen FR, et al. Antithrombotic drugs and subarachnoid haemorrhage risk. A nationwide case-control study in Denmark. Thromb Haemost. 2015; 114(5): 1064-1075, doi: 10.1160/TH15-04-0316, indexed in Pubmed: 26202836.

22. Starke RM, Chalouhi N, Ding D, et al. Potential role of aspirin in the prevention of aneurysmal subarachnoid hemorrhage. Cerebrovasc Dis. 2015; 39(5-6): 332-342, doi: 10.1159/000381137, indexed in Pubmed: 25967073.

23. Baharoglu MI, Schirmer CM, Hoit DA, et al. Aneurysm inflow-angle as a discriminant for rupture in sidewall cerebral aneurysms: morphometric and computational fluid dynamic analysis. Stroke. 2010; 41(7): 1423-1430, doi: 10.1161/STROKEAHA.109.570770, indexed in Pubmed: 20508183.

24. Tykocki T, Nauman P, Dow Enko A. Morphometric predictors of posterior circulation aneurysms risk rupture. Neurol Res. 2014; 36(8): 733-738, doi: $10.1179 / 1743132813 Y .0000000306$, indexed in Pubmed: 24620970.

25. Lv N, Feng Z, Wang C, et al. Morphological risk factors for rupture of small $(<7 \mathrm{~mm}$ ) posterior communicating artery aneurysms. World Neurosurg. 2016; 87: 311-315, doi: 10.1016/j.wneu.2015.12.055, indexed in Pubmed: 26724608.

26. Chiu JJ, Usami S, Chien S. Vascular endothelial responses to disturbed flow: pathologic implications for atherosclerosis. Bioengineering in Cell and Tissue Research. : 469-496, doi: 10.1007/978-3-54075409-1_19.

27. Ji W, Liu A, Lv X, et al. Larger inflow angle and incomplete occlusion predict recanalization of unruptured paraclinoid aneurysms after endovascular treatment. Interv Neuroradiol. 2016; 22(4): 383-388, doi: 10.1177/1591019916641315, indexed in Pubmed: 27066815.

28. Passerini AG, Polacek DC, Shi C, et al. Coexisting proinflammatory and antioxidative endothelial transcription profiles in a disturbed flow region of the adult porcine aorta. Proc Natl Acad Sci U S A. 2004; 101(8): 2482-2487, doi: 10.1073/pnas.0305938101, indexed in Pubmed: 14983035. 
29. Feng X, Zhang B, Guo E, et al. Bifurcation location and growth of aneurysm size are significantly associated with an irregular shape of unruptured intracranial aneurysms. World Neurosurg. 2017; 107: 255-262, doi: 10.1016/j.wneu.2017.07.063, indexed in Pubmed: 28735142.

30. Tremmel M, Dhar S, Levy El, et al. Influence of intracranial aneurysm-to-parent vessel size ratio on hemodynamics and implication for rupture: results from a virtual experimental study. Neurosurgery. 2009; 64(4): 622-30; discussion 630, doi: 10.1227/01. NEU.0000341529.11231.69, indexed in Pubmed: 19349824.

31. Ujiie $\mathrm{H}$, Tamano $\mathrm{Y}$, Sasaki $\mathrm{K}$, et al. Is the aspect ratio a reliable index for predicting the rupture of a saccular aneurysm? Neurosurgery. 2001; 48(3): 495-502; discussion 502, doi: 10.1097/00006123200103000-00007, indexed in Pubmed: 11270538.

32. Meng H, Tutino VM, Xiang J, et al. High WSS or low WSS? Complex interactions of hemodynamics with intracranial aneurysm initiation, growth, and rupture: toward a unifying hypothesis. AJNR Am J Neuroradiol. 2014; 35(7): 1254-1262, doi: 10.3174/ajnr.A3558, indexed in Pubmed: 23598838.

33. Zheng $\mathrm{Y}$, Zhou $\mathrm{B}$, Wang $\mathrm{X}$, et al. Size, aspect ratio and anatomic location of ruptured intracranial aneurysms: consecutive series of 415 patients from a prospective, multicenter, observational study.
Cell Transplant. 2018 [Epub ahead of print]: 963689718817227 , doi: 10.1177/0963689718817227, indexed in Pubmed: 30514102.

34. Amigo N, Valencia Á. Determining significant morphological and hemodynamic parameters to assess the rupture risk of cerebral aneurysms. Journal of Medical and Biological Engineering. 2018; 39(3): 329-335, doi: 10.1007/s40846-018-0403-0.

35. Mocco J, Brown RD, Torner JC, et al. International Study of Unruptured Intracranial Aneurysms Investigators. Aneurysm morphology and prediction of rupture: an international study of unruptured intracranial aneurysms analysis. Neurosurgery. 2018; 82(4): 491-496, doi: 10.1093/neuros/nyx226, indexed in Pubmed: 28605486.

36. Tykocki T, Kostkiewicz B. Correlation between the aneurysm morphometry and severity of subarachnoid hemorrhage in the posterior cerebral circulation. World Neurosurg. 2014; 82(6): 1100-1105, doi: 10.1016/j.wneu.2014.07.007, indexed in Pubmed: 25009163.

37. Wang AC, Heros RC. Editorial: Subarachnoid hemorrhage grading scales. J Neurosurg. 2016; 124(2): 296-298, doi: 10.3171/2015.3.JNS15336, indexed in Pubmed: 26381258.

38. Korja M, Kivisaari R, Rezai Jahromi B, et al. Size and location of ruptured intracranial aneurysms: consecutive series of 1993 hospital-admitted patients. J Neurosurg. 2017; 127(4): 748-753, doi: 10.3171/2016.9.JNS161085, indexed in Pubmed: 27911237. 